\title{
A note on intuitionistic fuzzy Menger spaces
}

\author{
A. Haydar Eș \\ Department of Mathematics Education, Başkent University \\ Bağlıca, 06490 Ankara, Turkey \\ e-mail: haydares@baskent.edu.tr
}

Received: 20 May 2020

Accepted: 8 September 2020

\begin{abstract}
In this paper, the concepts of intuitionistic fuzzy Mengerness, intuitionistic fuzzy near Mengerness and intuitionistic fuzzy almost Mengerness are introduced and studied. We give some characterizations of intuitionistic fuzzy almost Mengerness in terms of intuitionistic fuzzy regular open or intuitionistic fuzzy regular closed.
\end{abstract}

Keywords: Intuitionistic fuzzy topology, Intuitionistic fuzzy Menger spaces, Intuitionistic fuzzy near Menger spaces, Intuitionistic fuzzy almost Menger spaces.

202 Mathematics Subject Classification: 54A40, $03 E 72$.

\section{Introduction}

The concept of fuzzy set was introduced by Zadeh in his classic paper [14]. The concept of fuzzy topological spaces was introduced and developed by C. L. Chang [4]. In [7, 8] some weaker forms of fuzzy compactness are considered for the first time. Since Atanassov [1, 2, 3] introduced the notion of intuitionistic fuzzy sets, Çoker [5] defined the intuitionistic fuzzy topological spaces. Çoker and Eş [6] introduced and investigated fuzzy almost compactness, fuzzy near compactness and fuzzy light compactness in intuitionistic fuzzy topological spaces. The investigation of covering properties of topological spaces has a long history going back to papers by Menger and Rothberger [10, 12]. However, more recently a new theory called Selection Principles was introduced by Scheepers [13]. The theory of Selection Principles has extraordinary connections with numerous subareas of mathematics, for example, set theory and general topology, uniform structures and ditopological texture spaces [9]. In 1999, Kocinac defined and characterized the almost Menger property [9]. Following this concept, Aqsa, Moiz ud Din Khan defined and investigated nearly Menger and nearly star-Menger spaces [11].

In this paper, we introduce and then investigate intuitionistic fuzzy Menger spaces, Intuitionistic fuzzy near Menger spaces and Intuitionistic fuzzy almost Menger spaces in Çoker's space. 


\section{Preliminaries}

Definition 2.1. [1] Let $X$ be a non-empty fixed set and $I$ the closed interval [0, 1]. An intuitionistic fuzzy set (IFS) $A$ is an object of following form

$$
A=\left\{\left\langle x, \mu_{A}(x), \vartheta_{A}(x)\right\rangle \mid x \in X\right\} .
$$

where the mappings $\mu_{A}: X \rightarrow I$ and $\vartheta_{A}: X \rightarrow I$ denote the degree of membership $\mu_{A}(x)$ and the degree of non-membership $\vartheta_{A}(x)$ for each element $x \in X$ of the set $A$, respectively, and $0 \leq \mu_{A}(x)+\vartheta_{A}(x) \leq 1$ for each $x \in X$.

Definition 2.2. [1] Let $A$ and $B$ be IFS's of the form $A=\left\{\left\langle x, \mu_{A}(x), \vartheta_{A}(x)\right\rangle \mid x \in X\right\}$ and $B=\left\{\left\langle x, \mu_{B}(x), \vartheta_{B}(x)\right\rangle \mid x \in X\right\}$. Then
(i) $\quad A \subseteq B$ f $\mu_{A}(x) \leq \mu_{B}(x)$ and $\vartheta_{A}(x) \geq \vartheta_{B}(x)$
(ii) $\bar{A}=\left\{\left\langle x, \vartheta_{A}(x)(x), \mu_{A}(x)\right\rangle \mid x \in X\right\}$
(iii) $A \cap B=\left\{\left\langle x, \mu_{A}(x) \wedge \mu_{B}(x), \vartheta_{A}(x)(x) \vee \vartheta_{A}(x)(x)\right\rangle \mid x \in X\right\}$
(iv) $A \cup B=\left\{\left\langle x, \mu_{A}(x) \vee \mu_{B}(x), \vartheta_{A}(x)(x) \wedge \vartheta_{A}(x)(x)\right\rangle \mid x \in X\right\}$

Definition 2.3. [1] $0_{\sim}=\{\langle x, 0,1\rangle x \in X\}$ and $1_{\sim}=\{\langle x, 1,0\rangle x \in X\}$.

Definition 2.4. [5] An intuitionistic fuzzy topology (IFT for short) in Çoker's sense on a nonempty set $X$ is a family $\tau$ of intuitionistic fuzzy sets in $X$ satisfying the following axioms:

( $\left.\mathrm{T}_{1}\right) \quad 0_{\sim}, 1_{\sim} \in \tau$

$\left(\mathrm{T}_{2}\right) \quad G_{1} \cap G_{2} \in \tau$ for any $G_{1}, G_{2} \in \tau$,

( $\left.\mathrm{T}_{3}\right) \quad \bigcup_{i \in I} G_{i} \in \tau$ for any arbitrary family $\left\{G_{i}: i \in I\right\} \subseteq \tau$.

In this case the pair $(X, \tau)$ is called an intuitionistic fuzzy topological space (IFT for short) and each IFS in $\tau$ is known as an intuitionistic fuzzy open set (IFOS for short) in $X$.

Definition 2.5. [5] The complement $\bar{A}$ of an IFOS $A$ in an IFTS $(X, \tau)$ is called an intuitionistic fuzzy closed set (IFCS for short) in $X$.

Definition 2.6. [5] Let $(X, \tau)$ be an IFTS and $A=\left\{\left\langle x, \mu_{A}(x), \vartheta_{A}(x)\right\rangle \mid x \in X\right\}$ be an IFS in $X$. Then the fuzzy interior and fuzzy closure of $A$ are defined by

$$
\operatorname{cl}(A)=\cap\{K \mid K \text { is an IFCS in } X \text { and } A \subseteq K\}
$$

and

$$
\operatorname{int}(A)=\cup\{G \mid G \text { is an IFOS in } X \text { and } G \subseteq A\} .
$$

Definition 2.7. [5] Let $X$ and $Y$ be two nonempty sets and $f: X \rightarrow Y$ be a function. If $B=\left\{\left\langle y, \mu_{B}(y), \vartheta_{B}(y)\right\rangle \mid y \in Y\right\}$ is an IFS in $Y$, then the preimage of $B$ under $f$, denoted by $f^{-1}(B)$ is the IFS in $X$ defined by: 


$$
f^{-1}(B)=\left\{\left\langle x, f^{-1}\left(\mu_{B}\right)(x), f^{-1}\left(\vartheta_{B}\right)(x)\right\rangle \mid x \in X\right\} .
$$

Similarly, if $A=\left\{\left\langle x, \mu_{A}(x), \vartheta_{A}(x)\right\rangle \mid x \in X\right\}$ is an IFS in $X$, then the image of $A$ under $f$, denoted by $f(A)$, is the IFS in $Y$ defined by

$$
f(A)=\left\{\left\langle y, f\left(\mu_{A}\right)(y), f\left(\vartheta_{A}\right)(y)\right\rangle \mid y \in Y\right\} .
$$

Proposition 2.8. [5] Let $(X, \tau)$ be an IFTS and $A, B$ be IFS's in $X$. Then, the following properties hold:

$$
\begin{gathered}
\operatorname{cl}(\bar{A})=\overline{(\operatorname{int}(A))}, \operatorname{int}(\bar{A})=\overline{(\operatorname{cl}(A))} \\
\operatorname{int}(A) \subseteq A \subseteq \operatorname{cl}(A)
\end{gathered}
$$

Definition 2.9. [5] Let $X$ be an IFTS. A family $\left\{\left\langle x, \mu_{G_{i}}, \vartheta_{G_{i}}\right\rangle: i \in I\right\}$ of IFOS's in $X$ satisfies the condition $\bigcup\left\{\left\langle x, \mu_{G_{i}}, \vartheta_{G_{i}}\right\rangle: i \in I\right\}=1_{\sim}$ is called a fuzzy open cover of $X$.

A finite subfamily of a fuzzy open cover $\left\{\left\langle x, \mu_{G_{i}}, \vartheta_{G_{i}}\right\rangle: i \in I\right\}$ which is also a fuzzy open cover of $X$ is called a finite subcover of $\left\{\left\langle x, \mu_{G_{i}}, \vartheta_{G_{i}}\right\rangle: i \in I\right\}$. An IFTS $X$ is called fuzzy compact iff every fuzzy open cover has a finite subcover.

Definition 2.10. [6] Let $X$ be an IFTS. A family $\left\{\left\langle x, \mu_{G_{i}}, \vartheta_{G_{i}}\right\rangle: i \in I\right\}$ of IFOS's in $X$ has the finite intersection property (FIP for short) if every finite subfamily $\left\{\left\langle x, \mu_{G_{i}}, \vartheta_{G_{i}}\right\rangle: i=1,2, \ldots, n\right\}$ satisfies the condition $\bigcap_{i=1}^{n}\left\langle x, \mu_{G_{i}}, \vartheta_{G_{i}}\right\rangle \neq 0_{\sim}$.

Definition 2.11. [6] An intuitionistic fuzzy set $A$ is called an intuitionistic fuzzy regular open set iff $A=\operatorname{int}(\operatorname{cl}(A))$ an intuitionistic fuzzy set $B$ is called an intuitionistic fuzzy regular closed set iff $B=\operatorname{cl}(\operatorname{int}(B))$.

Definition 2.12. [6] Let $\left(X, \tau_{1}\right),\left(Y, \tau_{2}\right)$ be two IFTS's and let $f: X \rightarrow Y$ be a function. Then $f$ is said to be intuitionistic fuzzy strongly continuous iff for each IFS $A$ in $X, f(\operatorname{cl}(A)) \subseteq f(A)$.

Definition 2.13. [6] Let $\left(X, \tau_{1}\right)$ and $\left(Y, \tau_{2}\right)$ be two IFTS's and let $f: X \rightarrow Y$ be a function. Then $f$ is said to be intuitionistic fuzzy almost continuous iff the preimage of each intuitionistic fuzzy regular open set of $Y$ is an intuitionistic fuzzy open set in $X$.

Definition 2.14. [6] Let $\left(X, \tau_{1}\right)$ and $\left(Y, \tau_{2}\right)$ be two IFTS's and let $f: X \rightarrow Y$ be a function. Then $f$ is said to be intuitionistic fuzzy weakly continuous iff for each IFOS $B$ of $Y$, $f^{-1}(B) \subseteq \operatorname{int}\left(f^{-1}(\operatorname{cl}(B))\right)$. 


\section{Intuitionistic fuzzy Mengerness}

Here we generalize the concept of Menger topological spaces, first proposed by Menger [10], to the case of intuitionistic fuzzy topological spaces.

Definition 3.1 (i) An IFTS $(X, \tau)$ is fuzzy Menger, if for every sequence $\left\{G_{n} \mid n \in N\right\}$ where $G_{n}=\left\{\left\langle x, \mu_{G_{n}}, \vartheta_{G_{n}}\right\rangle \mid n \in N\right\}$ are intuitionistic fuzzy open covers of $X$, there exists a sequence $\left\{H_{n} \mid n \in N\right\}$, where $H_{n}=\left\{\left\langle x, \mu_{H_{n}}, \vartheta_{H_{n}}\right\rangle \mid n \in N\right\}$ such that for every $n \in N, H_{n}$ is a finite intuitionistic fuzzy subset of $G_{n}$ and $\cup\left\{\left\langle x, \mu_{H_{n}}, \vartheta_{H_{n}}\right\rangle \mid n \in N\right\}=1_{\sim}$.

(ii) An IFTS $(X, \tau)$ is fuzzy almost Menger (nearly Menger), if for every sequence $\left\{G_{n} \mid n \in N\right\}$ where $G_{n}=\left\{\left\langle x, \mu_{G_{n}}, \vartheta_{G_{n}}\right\rangle \mid n \in N\right\}$ are intuitionistic fuzzy open covers of $X$, there exists a sequence $\left\{H_{n} \mid n \in N\right\}$, where $H_{n}=\left\{\left\langle x, \mu_{H_{n}}, \vartheta_{H_{n}}\right\rangle \mid n \in N\right\}$ such that for every $n \in N, H_{n}$ is a finite intuitionistic fuzzy subset of $G_{n}$ and $\bigcup_{n \in N} H_{n}^{*}=1_{\sim}$, where $H_{n}^{*}=\left\{\operatorname{cl}(H) \mid H \subseteq H_{n}\right\}$ $\left(H_{n}^{*}=\left\{\operatorname{int}(\operatorname{cl}(H)) \mid H \subseteq H_{n}\right\}\right)$.

It is clear that in IFTS we have the following implications:

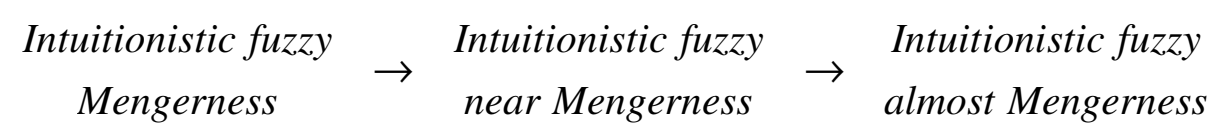

However, the reverse implications do not hold.

Example 3.2. Let $X=\{1,2\}$ and define the intuitionistic fuzzy subsets $\left\{G_{n} \mid n \in N\right\}$ as follows:

$$
\begin{array}{ll}
\mu_{G_{n}}(1)=1-\frac{1}{n}, & v_{G_{n}}(1)=\frac{1}{n+1}, \\
\mu_{G_{n}}(2)=1-\frac{1}{n+1}, & v_{G_{n}}(2)=\frac{1}{n+2} .
\end{array}
$$

In this case, the sequence $\tau=\left\{G_{n} \mid n \in N\right\} \cup\left\{0_{\sim}, 1_{\sim}\right\}$ is an IFT on $X$ [6]. Since $\operatorname{cl}\left(G_{n}\right)=1_{\sim}$ and $\operatorname{int}\left(\operatorname{cl}\left(G_{n}\right)\right)=1_{\sim},(X, \tau)$ is intuitionistic fuzzy nearly Menger. On the other hand, $\left\{G_{n} \mid n \in N\right\}$ has no finite intuitionistic fuzzy subset of $G_{n}$. Hence $(X, \tau)$ is not intuitionistic fuzzy Menger.

Theorem 3.3. An IFTS $(X, \tau)$ is fuzzy almost Menger iff every sequence $\left\{G_{n} \mid n \in N\right\}$, where $G_{n}=\left\{\left\langle x, \mu_{G_{n}}, \vartheta_{G_{n}}\right\rangle \mid n \in N\right\}$ of intuitionistic fuzzy open subset of $X$ having the finite intersection property we have $\bigcap_{n \in N} \operatorname{cl}\left(G_{n}\right) \neq 0_{\sim}$.

Proof. Suppose $X$ is fuzzy almost Menger and let $G_{n}=\left\{\left\langle x, \mu_{G_{n}}, \vartheta_{G_{n}}\right\rangle \mid n \in N\right\}$ be any sequence of intuitionistic fuzzy open sets in $X$ such that $\bigcap \operatorname{cl}\left\{\left\langle x, \mu_{G_{n}}, \vartheta_{G_{n}}\right\rangle \mid n \in N\right\}=0_{\sim}$. Then, we have $\bigcap \operatorname{int}\left\{\left\langle x, \mu_{G_{n}}, \vartheta_{G_{n}}\right\rangle \mid n \in N\right\}=1_{\sim}$. Since $X$ is fuzzy almost Menger, for every $n \in N$, there exists a 
sequence $\left\{H_{n} \mid n \in N\right\}$, where $H_{n}=\left\{\left\langle x, \mu_{H_{n}}, \vartheta_{H_{n}}\right\rangle \mid n \in N\right\}$, such that for every $n \in N, H_{n}$ is a finite intuitionistic fuzzy subset of $G_{n}$ and $\bigcup_{n \in N} H_{n}^{*}=1_{\sim}$, where $H_{n}^{*}=\left\{\operatorname{cl}(H) \mid H \subseteq H_{n}\right\}$. But from $H_{n} \subseteq \operatorname{int}\left(G_{n}^{c}\right)$ and $G_{n} \subseteq \operatorname{int}\left(\operatorname{cl}\left(G_{n}\right)\right)$, we see that $\bigcap_{n \in N} G_{n}=0_{\sim}$, which is a contradiction with the finite intersection property.

Conversely, let $\left\{G_{n} \mid n \in N\right\}$ be an intuitionistic fuzzy open cover of $X$. If $\bigcup_{n \in N} H_{n}^{*} \neq 1_{\sim}$, where $H_{n}^{*}=\left\{\operatorname{cl}(H) \mid H \subseteq H_{n}\right\}$ and $H_{n}$ is a finite intuitionistic fuzzy subset of $G_{n}$, then $\left(H_{n}^{*}\right)^{c}$ is an intuitionistic fuzzy open sequence with the finite intersection property. Hence, from the hypothesis it follows that $\bigcap_{n \in N} c l\left(\left(H_{n}^{*}\right)^{c}\right) \neq 0_{\sim}$ and then $\bigcup_{n \in N}\left[\operatorname{cl}\left(\left(H_{n}^{*}\right)^{c}\right)\right]^{c} \neq 1_{\sim}$. Since $\bigcup_{n \in N} G_{n} \subseteq \bigcup_{n \in N}\left[\operatorname{cl}\left(\left(H_{n}^{*}\right)^{c}\right)\right]^{c} \neq 1_{\sim}$, then $\bigcup_{n \in N} G_{n} \neq 1_{\sim}$, which is a contradiction.

Theorem 3.4. In an IFTS $(X, \tau)$ the following conditions are equivalent:

(i) $(X, \tau)$ is fuzzy almost Menger.

(ii) For every family $\left\{K_{n} \mid n \in N\right\}$, where $K_{n}=\left\{\left\langle x, \mu_{K_{n}}, \vartheta_{K_{n}}\right\rangle \mid n \in N\right\}$ of intuitionistic fuzzy regular closed sets such that $\bigcap_{n \in N} K_{n}=0_{\sim}$, there exists a sequence $\left\{H_{n} \mid n \in N\right\}$, where $H_{n}=\left\{\left\langle x, \mu_{H_{n}}, \vartheta_{H_{n}}\right\rangle \mid n \in N\right\}$, such that for every $n \in N, H_{n}$ is a finite subset of $K_{n}$ and $\bigcap_{n \in N} H_{n}^{*}=0_{\sim}$, where $H_{n}^{*}=\left\{\mathrm{cl}(H) \mid H \subseteq H_{n}\right\}$

(iii) $\bigcap_{n \in N} \operatorname{cl}\left(G_{n}\right) \neq 0_{\sim}$ holds for each sequence of intuitionistic fuzzy regular open sets $\left\{G_{n} \mid n \in N\right\}$ with the finite intersection property, where $G_{n}=\left\{\left\langle x, \mu_{G_{n}}, \vartheta_{G_{n}}\right\rangle \mid n \in N\right\}$.

(iv) For each sequence $\left\{G_{n} \mid n \in N\right\}$ of intuitionistic fuzzy regular open covers of $X$, there exists a sequence $\left\{H_{n} \mid n \in N\right\}$, where $H_{n}=\left\{\left\langle x, \mu_{H_{n}}, \vartheta_{H_{n}}\right\rangle \mid n \in N\right\}$ such that for $n \in N$, $H_{n}$ is a finite subset of $G_{n}$ and $\bigcup_{n \in N} H_{n}^{*}=1_{\sim}$, where $H_{n}^{*}=\left\{\operatorname{cl}(H) \mid H \subseteq H_{n}\right\}$.

Proof. The proof of this theorem follows a similar pattern to Theorem 3.3.

Theorem 3.5. Let $\left(X, \tau_{1}\right),\left(Y, \tau_{2}\right)$ be two IFTS's and let $f: X \rightarrow Y$ be an intuitionistic fuzzy almost continuous surjection. If $\left(X, \tau_{1}\right)$ is fuzzy almost Menger, then $\left(Y, \tau_{2}\right)$ is fuzzy almost Menger.

Proof. Let $\left\{G_{n} \mid n \in N\right\}$ be a sequence of covers of $Y$ by fuzzy open sets, then $\left\{\operatorname{int}\left(\operatorname{cl}\left(G_{n}\right)\right) \mid n \in N\right\}$ is also an intuitionistic fuzzy open cover of $Y$. Since $f$ is fuzzy almost continuous, $\left\{f^{-1}\left(\operatorname{int}\left(\operatorname{cl}\left(G_{n}\right)\right)\right) \mid n \in N\right\}$ is an intuitionistic fuzzy open cover of $X$. Thus, there is a sequence $\left\{H_{n} \mid n \in N\right\}$, where $H_{n}=\left\{\left\langle x, \mu_{H_{n}}, \vartheta_{H_{n}}\right\rangle \mid n \in N\right\}$, such that for every $n \in N, H_{n}$ is a finite subset of $f^{-1}\left(\operatorname{int}\left(\operatorname{cl}\left(G_{n}\right)\right)\right)$ and $\bigcup_{n \in N} H_{n}^{*}=1_{\sim}$, where $H_{n}^{*}=\left\{\operatorname{cl}(H) \mid H \subseteq H_{n}\right\}$. 
For every $n \in N$ and $H \subseteq H_{n}$ we can choose a member $G_{H} \subseteq G_{n}$ such that $H=f^{-1}\left(G_{H}\right)$. From the surjectivity of $f$ we have

$$
f\left(\bigcup_{n \in N} \operatorname{cl}\left(f^{-1}\left(\operatorname{int}\left(\operatorname{cl}\left(G_{H}\right)\right)\right)\right)\right)=\bigcup_{n \in N} f\left(\operatorname{cl}\left(f^{-1}\left(\operatorname{int}\left(\operatorname{cl}\left(G_{H}\right)\right)\right)\right)\right)=1_{\sim} .
$$

But from int $\left(\operatorname{cl}\left(G_{H}\right)\right) \subseteq \operatorname{cl}\left(G_{H}\right)$ and intuitionistic fuzzy almost continuity of $f, f^{-1}\left(\operatorname{cl}\left(G_{H}\right)\right)$ must be an intuitionistic fuzzy closed set containing $f^{-1}\left(\operatorname{int}\left(\operatorname{cl}\left(G_{H}\right)\right)\right)$ and then $\operatorname{cl}\left(f^{-1}\left(\operatorname{int}\left(\operatorname{cl}\left(G_{H}\right)\right)\right)\right)$. Therefore, $f\left(\operatorname{cl}\left(f^{-1}\left(\operatorname{int}\left(\operatorname{cl}\left(G_{H}\right)\right)\right)\right)\right) \subseteq \operatorname{cl}\left(G_{H}\right)$.

Then, $\bigcup_{n \in N}\left\{\operatorname{cl}\left(G_{H}\right) \mid H \subseteq H_{n}\right\}=1_{\sim}$. Hence, $Y$ is intuitionistic fuzzy almost Menger.

Theorem 3.6. Let $\left(X, \tau_{1}\right),\left(Y, \tau_{2}\right)$ be two IFTS's and let $f: X \rightarrow Y$ be an intuitionistic fuzzy strongly continuous surjection. If $\left(X, \tau_{1}\right)$ is fuzzy almost Menger, then so is $\left(Y, \tau_{2}\right)$. Proof. It is similar to the proof of Theorem 3.5.

Theorem 3.7. Let $\left(X, \tau_{1}\right),\left(Y, \tau_{2}\right)$ be two IFTS's and let $f: X \rightarrow Y$ be an intuitionistic fuzzy weakly fuzzy continuous surjection. If $\left(X, \tau_{1}\right)$ is fuzzy Menger, then $\left(Y, \tau_{2}\right)$ is fuzzy almost Menger.

Proof. The proof follows from the definition of intuitionistic fuzzy weakly continuous function.

Theorem 3. 8. An intuitionistic fuzzy topological space $(X, \tau)$ is intuitionistic fuzzy nearly Menger iff for every sequence $\left\{G_{n} \mid n \in N\right\}$, where $G_{n}=\left\{\left\langle x, \mu_{G_{n}}, \vartheta_{G_{n}}\right\rangle \mid n \in N\right\}$ of covers of $X$ by intuitionistic fuzzy regular open sets, there is a sequence $\left\{H_{n} \mid n \in N\right\}$, where $H_{n}=$ $\left\{\left\langle x, \mu_{H_{n}}, \vartheta_{H_{n}}\right\rangle \mid n \in N\right\}$, such that for every $n \in N, H_{n}$ is a finite subset of $G_{n}$ and $\bigcup_{n \in N} H_{n}=1_{\sim}$.

Proof. Similar to the proof of Theorem 3.3.

\section{References}

[1] Atanassov K. T. (1983). Intuitionistic Fuzzy Sets, VII ITKR Session, Sofia, 20-23 June 1983 (Deposed in Centr. Sci.-Techn. Library of the Bulg. Acad. of Sci., 1697/84) (in Bulgarian). Reprinted: (2016) Int. J. Bioautomation, 20(S1), S1-S6 (in English).

[2] Atanassov, K. T., \& Stoeva, S. (1984). Intuitionistic L-fuzzy sets, Cybernetics and System Research, 2, 539-540.

[3] Atanassov, K. T. (1986). Intuitionistic fuzzy sets, Fuzzy Sets and Systems, 20 (1), 87-96.

[4] Chang, C. L. (1968). Fuzzy topological spaces, J. Math. Anal., 24, 182-190.

[5] Çoker, D. (1997). An introduction to intuitionistic fuzzy topological spaces, Fuzzy Sets and Systems, 88, 81-89. 
[6] Çoker, D., \& Eş, A. H. (1995). On fuzzy compactness in intuitionistic fuzzy topological spaces, The Journal of Fuzzy Mathematics, 3, 899-909.

[7] Eş, A. H. (1987). Almost compactness and near compactness in fuzzy topological spaces, Fuzzy Sets and Systems, 22, 289-295.

[8] Concilio, A. Di, \& Gerla, G. (1984). Almost compactness in fuzzy topological spaces, Fuzzy Sets and Systems, 13, 184-192.

[9] Kocinac, Lj. D. R. (1999). Star-Menger and related spaces II, Filomat, 13, 129-140.

[10] Menger, K. (1924). Einige Überdeckungssatze der Punktmengenlehre, Sitzungsberichte Abt. 2a, Mathematik, Astronomie, Physik, Meteorologie und Mechanik. Wiener Akademie, Wien, 133, 421-444.

[11] Parvez, A., \& Khan, M. (2019). On Nearly Menger and Nearly Star-Menger Spaces, Filomat, 33 (19), 6219-6227.

[12] Rothberger, F. (1938). Eine Verscharfung dar Eigenschaft G, Fund. Math., 30, 50-55.

[13] Scheepers, M. (1996). Combinatorics of open covers I: Ramsey Theory, Topology Appl., 73, 241-266.

[14] Zadeh, L. A. (1965). Fuzzy sets, Information and Control, 8, 338-353. 